\title{
High incidence and mortality of Pneumocystis jirovecii infection in anti- MDA5-antibody-positive dermatomyositis: experience from a single center
}

\author{
Linlin Huang, Qiong Fu, Yan Ye, Yanwei Lin, Qingran Yan ${ }^{*+}$ and Sheng Chen ${ }^{*+}$ (D)
}

\begin{abstract}
Background: Idiopathic inflammatory myopathies (IIM) are associated with a significantly higher risk of opportunistic infections including Pneumocystis jirovecii pneumonia (PJP), a potentially fatal opportunistic infection. However, no prior studies have evaluated PJP infection in subtypes of IIM.

Objectives: To investigate the prevalence and mortality rate of PJP infection in subgroups of IIM patients stratified according to myopathy-specific antibodies.

Methods: In the first part of the study, 463 consecutive patients with IIM were prospectively followed for a period of at least 1 year to analyze the incidence of PJP. In the second part of the study, we enrolled 30 consecutive PJP patients with any rheumatic disease in order to identify the mortality rate and risk factors by Cox regression analysis. The Kaplan-Meier method with log-rank testing was used to assess differences in survival.

Results: The prevalence of PJP in IIM patients was found to be 3.0/100 person-years, while in MDA5 ${ }^{+}$DM patients it was 7.5/100 person-years and in MDA5 ${ }^{-} \| \mathrm{M}$ patients $0.7 / 100$ person-years $(P<0.05)$. PJP typically occurred in the first 2 months in the case of MDA5 $5^{+} \mathrm{DM}$ patients who had a significant decrease in their $\mathrm{CD} 4^{+} \mathrm{T}$ cell counts and lymphocyte counts $(P<0.05)$. In PJP patients, 3-month mortality was higher for MDA5 $5^{+}$DM patients than in those with other rheumatic diseases $(83.3 \%$ vs $38.9 \%, P<0.05)$. Alarmingly, $\mathrm{MDA}^{+}{ }^{+} \mathrm{DM}$ patients seemed not to benefit from prompt anti-PJP treatment, unlike patients with other rheumatic diseases whose survival improved when antiPJP treatment was started within 6 days $(P<0.05)$.
\end{abstract}

Conclusion: PJP has an alarming high incidence and mortality in $\mathrm{MDA}^{+} \mathrm{DM}$ patients. Timely treatment for PJP seems not to improve the prognosis of patients with this particular subtype. Hence, there remains a crucial unmet need to develop PJP prophylaxis for MDA5 ${ }^{+}$DM patients.

Keywords: Anti-MDA5-antibody-positive dermatomyositis, Pneumocystis jirovecii pneumonia, Incidence, Mortality, Treatment

\footnotetext{
* Correspondence: Yanqingran@163.com; 13917556052@139.com

${ }^{\dagger}$ Qingran Yan and Sheng Chen contributed equally to this work. Department of Rheumatology, Renji Hospital, Shanghai Jiao Tong University School of Medicine, Shanghai 200001, China
}

C C The Author(s). 2021 Open Access This article is licensed under a Creative Commons Attribution 4.0 International License, which permits use, sharing, adaptation, distribution and reproduction in any medium or format, as long as you give appropriate credit to the original author(s) and the source, provide a link to the Creative Commons licence, and indicate if changes were made. The images or other third party material in this article are included in the article's Creative Commons licence, unless indicated otherwise in a credit line to the material. If material is not included in the article's Creative Commons licence and your intended use is not permitted by statutory regulation or exceeds the permitted use, you will need to obtain permission directly from the copyright holder. To view a copy of this licence, visit http://creativecommons.org/licenses/by/4.0/. The Creative Commons Public Domain Dedication waiver (http://creativecommons.org/publicdomain/zero/1.0/) applies to the data made available in this article, unless otherwise stated in a credit line to the data. 


\section{Key messages}

1. The incidence of PJP in IIM patients is significantly higher in $\mathrm{MDA}^{+}$than $\mathrm{MDA} 5^{-}$subtype.

2. The PJP mortality of MDA5 ${ }^{+} \mathrm{DM}$ patients is higher than that in patients with other rheumatic diseases.

3. Timely anti-PJP treatment significantly improves the prognosis of PJP in rheumatic disease yet has no benefit for MDA $5^{+} \mathrm{DM}$ patients.

4. Our data suggest the necessity of developing PJP prophylaxis in MDA5 $5^{+} \mathrm{DM}$ patients, especially in the first 3 months of treatment or when the patient's $\mathrm{CD} 4^{+} \mathrm{T}$ cell count decreases to $<200$ cells/ $\mu \mathrm{L}$.

\section{Introduction}

Patients with rheumatic disease receiving intensive immunosuppressive therapy, and who are therefore immunocompromised, often suffer from opportunistic infections [1]. The risk of opportunistic infection is highest for dermatomyositis/polymyositis (PM/DM) patients, followed by systemic lupus erythematosus, systemic sclerosis, rheumatoid arthritis, and finally primary Sjogren's syndrome [2]. A recent study showed that underlying PM/DM significantly predisposes patients to Pneumocystis jirovecii pneumonia (PJP) [3]. This is a rare but potentially life-threatening opportunistic infection with a $30-60 \%$ mortality rate among immunocompromised (non-HIV) patients $[4,5]$. In patients with rheumatic immune diseases, most PJP occurs in the first 3 months after initiating immunosuppressive therapy $[2$, 6].

The term idiopathic inflammatory myopathy (IIM) denotes a group of autoimmune diseases characterized by myasthenia and typical skin rash, among which PM and DM are the most common. Myositis-specific antibodies have long been identified and their value for stratifying patients with different outcomes has been recognized. However, there are very few reports on PJP in the different IIM subtypes. A recent study identified antimelanoma differentiation-associated gene 5 antibody (anti-MDA5) as the only myositis-specific antibody that was associated with PJP in a multicenter juvenile DM cohort [7].

Unfortunately, MDA5 ${ }^{+} \mathrm{DM}$ is one of the subtypes of IIM with a poor prognosis and is mainly characterized by progressive interstitial lung disease, with or without muscle damage [8]. For adult $\mathrm{MDA}^{+}$patients, there appears to be only one report on two PJP+ deceased cases [9]. Hence, the mortality rate for PJP in $\mathrm{MDA}^{+}$patients is unknown. Therefore, in the present study, we first investigated the incidence of PJP in IIM patients with or without anti-MDA5 antibody and then analyzed the outcomes of anti-PJP treatment and mortality risk factors for PJP infection in different rheumatic diseases.

\section{Patients and methods Patients}

In the first part of the study, we evaluated PJP incidence in an IIM cohort. All clinically diagnosed adult IIM patients fulfilling the 1997 classification criteria [10] were prospectively recruited from May 2017 to January 2020 at the Department of Rheumatology, Renji Hospital, China. Patients were screened for myositis-specific antibodies and myositis-associated antibodies using a commercial immunoblot assay with 16 autoantigens. Baseline characteristics of patients in the hospital, including demographic, clinical, and laboratory data, were acquired from the patients' electronic medical records. Follow-up data were collected over a period of at least 1 year. The occurrence of PJP was evaluated in these patients.

In the second part of the study, anti-PJP treatment outcomes were evaluated in all PJP patients with rheumatic diseases. Thirty adult PJP patients with rheumatic diseases were recruited in a consecutive cohort study from May 2017 to January 2020 at the Department of Rheumatology, Renji Hospital, once the diagnosis of PJP was confirmed. Baseline characteristics including demographic, clinical, and laboratory data were acquired at the time of the patient's first admission to the hospital. Follow-up data were collected over a period of at least 3 months. The 3-month cumulative survival rates were then evaluated. Written informed consent was obtained from each study participant. The study was conducted in accordance with the Declaration of Helsinki and was approved by the Ethics Committee of Renji Hospital, Shanghai, China (ID: 2013-126).

\section{Diagnosis of PJP}

The diagnosis of PJP was based on comprehensive evaluation by clinical manifestations such as fever or acute dyspnea, characteristic radiographic findings, and etiological evidence. For confirmation, a case needed to have positive microbiological tests such as by next-generation sequencing and Grocott-Gomori methenamine-silver staining of bronchoalveolar lavage fluid. A probable case with typical manifestations but no etiological evidence needed confirmation by two infection specialists. However, a positive PJP sequencing result in the absence of clinical manifestations was not sufficient to define PJP infection.

\section{Statistical analysis}

Statistical analysis was performed using the SPSS 23.0 software package (IBM Corp., Armonk, NY, USA) and GraphPad Prism 8.0 (GraphPad Software). We used the 
chi-square test or Fisher's exact test to compare categorical variables and Student's $t$ test or the Mann-Whitney $U$ test to compare continuous variables. Logistic regression was performed for multivariable analysis to identify independent risk factors for PJP occurrence and to calculate odds ratios. Cox proportional hazards regression was performed as a multivariable analysis to identify independent risk factors for death and to calculate hazard ratios. The optimal cut-off value was determined by using the receiver operating characteristic (ROC) analysis and the Kaplan-Meier method with log-rank testing was employed to assess differences in survival. For all analyses, two-tailed $P$-values less than 0.05 were considered statistically significant.

\section{Results}

\section{PJP incidence in IIM patients}

There were 14 PJP patients in the IIM cohort $(n=463)$ after at least a 1-year follow-up (median follow-up 18 months, range 1-42 months). We calculated the prevalence of PJP in the IIM cohort at 3.0/100 person-years, while in $\mathrm{MDA}^{+} \mathrm{DM}$ patients, this rose to $7.5 / 100$ person-years, in contrast to MDA5 $5^{-}$IIM patients where it was only $0.7 / 100$ person-years (Fig. $1,7.5 \%$ vs $0.7 \%, P$ $<0.00001$ ). IIM patients were stratified into a $\mathrm{PJP}^{+}$group and a PJP $\mathrm{P}^{-}$group to assess risk factors for PJP infection (Table 1). We found a significant imbalance in the distribution of anti-MDA5 positivity, which was higher in

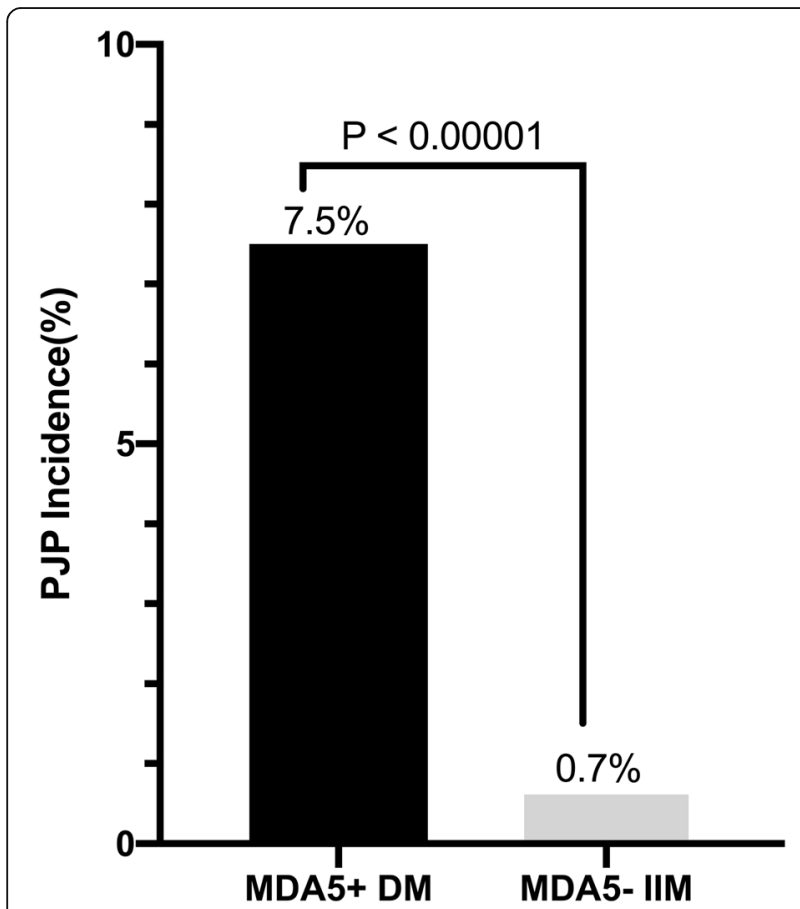

Fig. 1 One-year PJP incidence in IIM patients. The incidence of MDA5 ${ }^{+} \mathrm{DM}$ is $7.5 / 100$ person-years, while MDA5 $5^{-} \| \mathrm{M}$ is $0.7 / 100$ person-years. IIM, idiopathic inflammatory myopathy
PJP $^{+}$IIM patients $(85.7 \%$ vs $33.0 \%, P<0.0001)$. In addition to clinical factors such as a shorter median course ( 2 months vs 6 months), interstitial lung disease (92.9\% vs $70.4 \%)$, presence of diabetes (42.9\% vs $13.6 \%)$, and higher prednisone exposure $(50 \mathrm{mg}$ vs $30 \mathrm{mg}$ ), we also found that laboratory parameters such as higher erythrocyte sedimentation rate and ferritin value, lower serum albumin, $\mathrm{CD} 4^{+} \mathrm{T}$ lymphocyte count, and overall lymphocyte count were all significantly different between PJP $^{-}$and PJP ${ }^{+}$IIM patients $(P<0.05)$.

Considering the poor prognosis of $\mathrm{MDA}^{+} \mathrm{DM}$, the requirement for aggressive treatment may blur the identification of risk factors for PJP infection. As shown in Table S1 (online), $\mathrm{MDA}^{+} \mathrm{DM}$ patients in this cohort did receive higher doses of corticosteroids and much more immunosuppression (IS) using agents such as cyclosporine, tacrolimus, and biologics. To identify risk factors, we used a logistic regression model and included factors with $P$ values $<0.15$ in dichotomous comparisons between $\mathrm{PJP}^{+}$and PJP ${ }^{-}$IIM patients, such as corticosteroid dose, tacrolimus use, $\mathrm{CD} 4^{+} \mathrm{T}$ cell counts, albumin level, presence of diabetes, $\mathrm{MDA}^{+} \mathrm{DM}$, disease duration, and interstitial lung disease. As shown in Table 2, only the presence of anti-MDA5 antibody and low $\mathrm{CD} 4^{+}$ $\mathrm{T}$ cell counts were identified as independent risk factors for PJP occurrence by this multivariate analysis.

In an additional comparison between $\mathrm{PJP}^{-}$and $\mathrm{PJP}^{+}$ patients with similar treatment and diabetes status among the $\mathrm{MDA}^{+} \mathrm{DM}$ patients (Table S2), PJP occurred at a median of 2 months and at the time of a clear decrease of $\mathrm{CD}_{4}^{+} \mathrm{T}$ cell count and lymphocyte count.

\section{PJP infection in cohorts with rheumatic diseases}

To evaluate the impact of anti-MDA5 positivity on mortality caused by PJP, we further analyzed all $\mathrm{PJP}^{+}$patients $(n=30)$ admitted to our medical center during the same period as the IIM cohort. Clinical features compared between $M D A 5^{+}$DM patients and those with other rheumatic diseases are shown in Supplementary Table S3 (online), with more details of the $30 \mathrm{PJP}^{+} \mathrm{pa}-$ tients provided in Supplementary Table S4 (online).

As shown in Fig. 2A, MDA5 ${ }^{+} \mathrm{DM}$ constituted the greater part of the $\mathrm{PJP}^{+}$patients $(n=12,39.60 \%)$, followed by systemic lupus erythematosus (7, 22.70\%), MDA5 ${ }^{-}$IIM (2, 6.93\%), ANCA-associated vasculitis (AAV; 2, 6.93\%), adult-onset Still's disease (AOSD; 2, 6.93\%), primary Sjogren's syndrome (pSS; 2, 6.93\%), undifferentiated connective tissue disease (UCTD; 2, 6.93\%), and finally, a single patient with rheumatoid arthritis (RA; 2.97\%). The heatmap in Fig. 2B shows the duration of rheumatic disease at the time that PJP infection occurred. For IIM, AAV, and AOSD patients, PJP infection mostly occurred within 6 months of disease 
Table 1 Comparison of risk factors in PJP and non-PJP cases for IIM patients

\begin{tabular}{|c|c|c|c|}
\hline & PJP $(n=14)$ & Non-PJP $(n=449)$ & $P$ value \\
\hline $\mathrm{MDA}^{+} \mathrm{DM}, n(\%)$ & $12(85.7 \%)$ & $148(33.0 \%)$ & 0.000 \\
\hline Non-MDA5 ॥M, n (\%) & $2(14.3 \%)$ & $301(67.0 \%)$ & \\
\hline Male gender, $n(\%)$ & $6(42.9 \%)$ & $135(30.1 \%)$ & 0.376 \\
\hline Assessed age, mean \pm SD & $54 \pm 10$ & $53 \pm 12$ & 0.635 \\
\hline Disease duration, years, median & 2 & 6 & 0.001 \\
\hline ILD (\%) & $13(92.9 \%)$ & $316(70.4 \%)$ & 0.077 \\
\hline \multicolumn{4}{|l|}{ Premedication (last 1 month), $n$ (\%) } \\
\hline Corticosteroid ( $\geq 20 \mathrm{mg}$ pred, $\geq 1$ month) & $10(71.4 \%)$ & $226(50.4 \%)$ & 0.12 \\
\hline Corticosteroid, mg, median & 50 & 30 & 0.001 \\
\hline Cyclophosphamide & $2(14.3 \%)$ & $33(7.3 \%)$ & 0.286 \\
\hline Methotrexate & $0(0 \%)$ & $37(8.3 \%)$ & 0.616 \\
\hline Azathioprine & $0(0 \%)$ & $32(7.1 \%)$ & 0.613 \\
\hline Cyclosporine & $3(21.4 \%)$ & 69 (15.4\%) & 0.465 \\
\hline Tacrolimus & $4(28.6 \%)$ & $50(11.1 \%)$ & 0.068 \\
\hline Mycophenolate mofetil & $0(0 \%)$ & $24(5.3 \%)$ & 1.000 \\
\hline Hydroxychloroquine & $4(28.6 \%)$ & 95 (21.2\%) & 0.511 \\
\hline Biologics & $1(7.1 \%)$ & $16(3.6 \%)$ & 0.412 \\
\hline Others & $1(7.1 \%)$ & 76 (16.9\%) & 0.482 \\
\hline Diabetes, n (\%) & $6(42.9 \%)$ & $61(13.6 \%)$ & 0.009 \\
\hline ESR, mm/h, median & 34.5 & 21 & 0.085 \\
\hline Creatine kinase, $U / L$, median & 26.5 & 67.5 & 0.02 \\
\hline $\mathrm{LDH}, \mathrm{U} / \mathrm{L}$, median & 464.5 & 321 & 0.028 \\
\hline Ferritin, $\mu \mathrm{g} / \mathrm{mL}$, median & 1122 & 341 & 0.001 \\
\hline Pre-albumin, $g / L$, median & 208.5 & 214 & 0.832 \\
\hline Albumin, mg/L, median & 29.4 & 32.5 & 0.005 \\
\hline $\mathrm{CD}^{+} \mathrm{T}$ cell counts at admission $\times 10^{9} / \mathrm{L}$, median & 113.4 & 350.4 & 0.000 \\
\hline Lymphocyte counts at admission $\times 10^{9} / L$, median & 0.695 & 0.9 & 0.004 \\
\hline
\end{tabular}

ILD interstitial lung disease, ESR erythrocyte sedimentation rate, $L D H$ lactic dehydrogenase, $C K$ creatine kinase

onset, while a little unexpectedly for SLE patients, PJP seemed to occur at any stage of disease in this cohort.

\section{PJP mortality}

The mortality of anti-MDA5 antibody-positive patients was higher than that in other rheumatic diseases $(83.3 \%$ vs $38.9 \% P=0.016$ ), as shown in Fig. $2 \mathrm{C}$ and $\mathrm{D}$. We identified age, $\mathrm{CD} 4^{+} \mathrm{T}$ cell counts, and $\mathrm{MDA}^{+} \mathrm{DM}$ as risk factors for 3 -month mortality at $P<0.10$ by univariate analysis. These 3 factors were then analyzed in the Cox regression model, which confirmed that MDA5 ${ }^{+}$ $\mathrm{DM}$ and $\mathrm{CD}_{4}^{+} \mathrm{T}$ cell counts were independent risk factors for mortality. Notably, of these two variables, despite having been previously identified as PJP mortality factors [11], $\mathrm{CD}_{4}^{+} \mathrm{T}$ cell counts only yielded a hazard ratio of 0.994 (95\% CI $0.989-1.000)$ while $\mathrm{MDA}^{+} \mathrm{DM}$ had a much higher HR of 3.254 (95\% CI 1.209-8.756) (Table 3).
In addition to baseline risk factors, prompt anti-PJP treatment is a critical influence on patient survival. It has long been recognized that early diagnosis and treatment can improve the prognosis of PJP patients $[12,13]$. We sought the relevant time limitation for optimal PJP treatment using ROC analysis, but the best cut-off value was not statistically significant (Supplementary Figure S1A, $P=0.0983$, at a cut-off value of 7 days). When we further stratified all patients by anti-MDA5 status, a similar ROC as seen in previous reports was obtained for patients with rheumatic diseases other than MDA $5^{+}$ DM. The time-to-PJP-treatment cut-off of 6 days exhibited an $85.7 \%$ sensitivity and $63.6 \%$ specificity, with an area under the curve (AUC) of $81.2 \%$ by ROC analysis. Thus, the time of 6 days was the optimal cut-off point for timely treatment of PJP (Supplementary Figure S1B, $P=0.0297)$. However, in $\mathrm{MDA}^{+} \mathrm{DM}$ patients, ROC analysis was uninformative (Figure S1C). We then 
Table 2 Risk factors for PJP occurrence in IIM patients

\begin{tabular}{|c|c|c|c|c|}
\hline \multirow[t]{2}{*}{ Variable } & \multicolumn{2}{|l|}{ Univariable } & \multicolumn{2}{|l|}{ Multivariable } \\
\hline & OR $(95 \% \mathrm{Cl})$ & $P$ value & OR $(95 \% \mathrm{Cl})$ & $P$ value \\
\hline Corticosteroid dose, mg & $1.005(0.999,1.012)$ & 0.117 & - & 0.703 \\
\hline Corticosteroid ( $\geq 20 \mathrm{mg}, \geq 1$ month) & $1.76(0.581,5.335)$ & 0.317 & * & * \\
\hline Diabetes & $4.758(1.596,14.186)$ & 0.005 & - & 0.067 \\
\hline Tacrolimus & $2.865(0.869,9.451)$ & 0.084 & - & 0.616 \\
\hline Mycophenolate mofetil & $1.157(0.146,9.162)$ & 0.890 & * & * \\
\hline Ciclosporin & $2.027(0.619,6.637)$ & 0.243 & * & * \\
\hline Cyclophosphamide & $0.809(0.103,6.347)$ & 0.840 & * & * \\
\hline Methotrexate & $0.000(0.000,0.000)$ & 0.998 & * & * \\
\hline Biological agent & $0.000(0.000,0.000)$ & 0.999 & * & * \\
\hline $\mathrm{MDA}^{+} \mathrm{DM}$ & $12.203(2.696,55.229)$ & 0.001 & $6.374(1.368,29.722)$ & 0.018 \\
\hline Age & $1.006(0.963,1.050)$ & 0.790 & * & * \\
\hline Interstitial lung disease & $5.472(0.709,42.249)$ & 0.103 & - & 0.742 \\
\hline Erythrocyte sedimentation rate & $1.001(0.997,1.005)$ & 0.659 & * & * \\
\hline Lactic dehydrogenase & $1.000(1.000,1.001)$ & 0.269 & * & * \\
\hline CD4 $+T$ cell counts at admission & $0.991(0.985,0.996)$ & 0.001 & $0.986(0.992,0.997)$ & 0.003 \\
\hline Lymphocyte counts at admission & $0.099(0.019,0.522)$ & 0.006 & - & 0.589 \\
\hline Disease duration, months, median & $0.763(0.600,0.970)$ & 0.027 & - & 0.066 \\
\hline Albumin & $0.844(0.761,0.937)$ & 0.001 & - & 0.667 \\
\hline
\end{tabular}

$\mathrm{Cl}$ confidence interval

*Not included in the multivariable model due to the lack of significant association in the univariable analysis

- Included in the multivariable model but lacking of significant association in the multivariate analysis

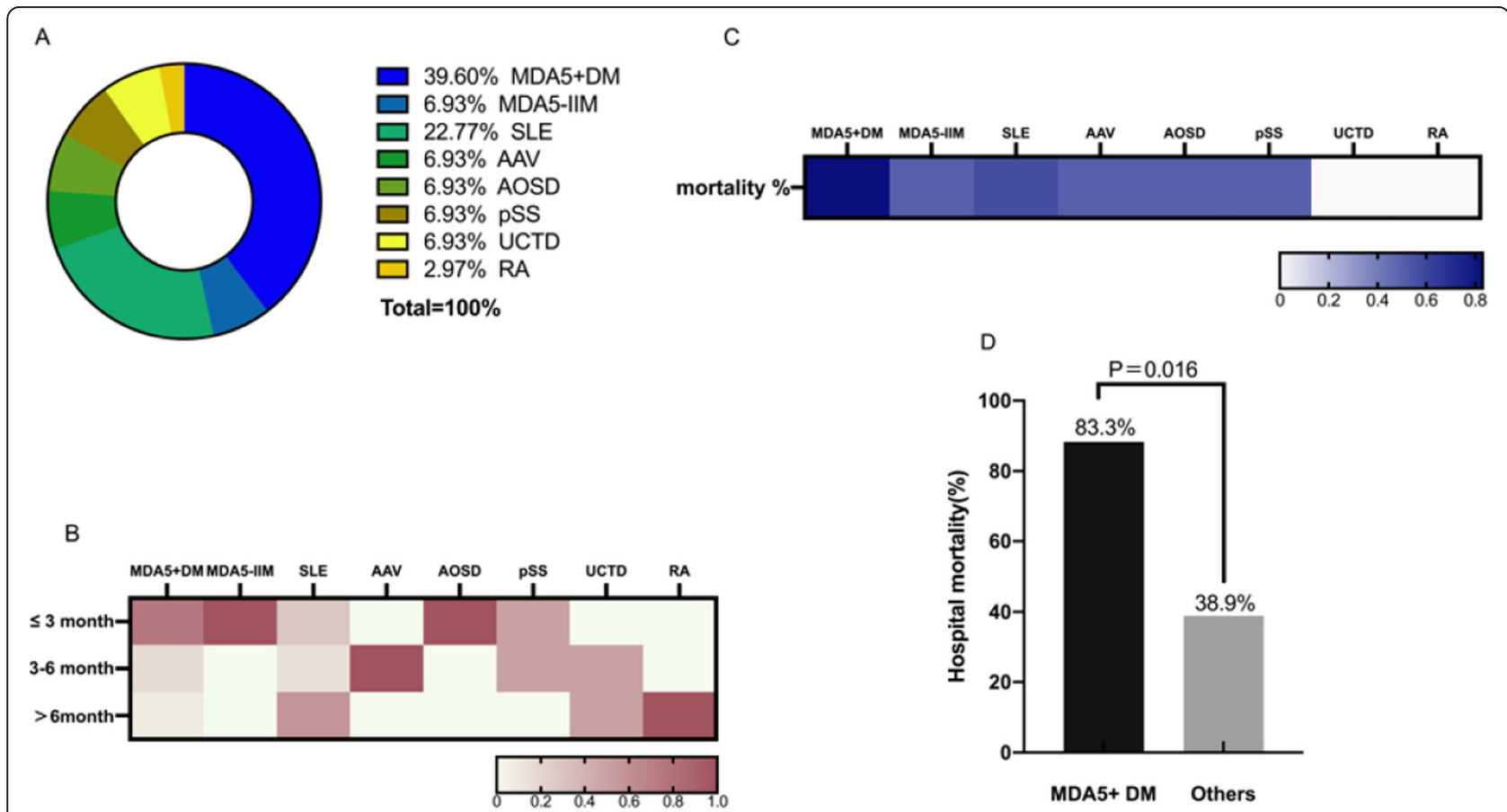

Fig. 2 The clinical characters and mortality of the PJP cohort. This cohort contains all PJP cases from our center in the recent 3 years. A The distribution of underlying rheumatic diseases in all $30 \mathrm{PJP}+$ patients in this cohort. B The distribution of rheumatic disease duration at PJP onset. C The mortality of PJP cases in each rheumatic disease. $\mathbf{D}$ Comparison of PJP-associated mortality in MDA5 ${ }^{+}$DM and other rheumatic diseases 
Table 3 Risk factors for 3-month mortality of PJP with rheumatic disease

\begin{tabular}{|c|c|c|c|c|}
\hline \multirow[b]{2}{*}{ Clinical factors } & \multicolumn{2}{|l|}{ Univariable } & \multicolumn{2}{|l|}{ Multivariable } \\
\hline & HR $(95 \% \mathrm{Cl})$ & $P$ value & HR $(95 \% \mathrm{Cl})$ & $P$ value \\
\hline Assessed age & $0.981(0.951-1.012)$ & 0.225 & $0.975(0.940-1.010)$ & 0.158 \\
\hline Gender & $0.437(0.207-0.923)$ & 0.798 & * & * \\
\hline $\mathrm{MDA}^{+} \mathrm{DM}$ & $2.830(1.067-7.505)$ & 0.037 & 3.254 (1.209-8.756) & 0.02 \\
\hline AAV & $0.674(0.089,5.099)$ & 0.702 & * & * \\
\hline SLE & $1.062(0.346,3.265)$ & 0.916 & * & * \\
\hline $\mathrm{CD}^{+} \mathrm{T}$ cell counts & $0.995(0.989-1.001)$ & 0.083 & $0.994(0.989-1.000)$ & 0.04 \\
\hline Lymphocyte counts & $0.142(0.025-0.808)$ & 0.028 & * & * \\
\hline Interstitial lung disease & $1.537(0.540-4.375)$ & 0.421 & * & * \\
\hline Steroid dose & $0.291(0.063,1.350)$ & 0.115 & * & * \\
\hline Cyclophosphamide & $1.807(0.586,5.568)$ & 0.303 & * & * \\
\hline Rituximab & $0.854(0.113,6.450)$ & 0.878 & * & * \\
\hline Time to anti-PJP treatment & $1.577(0.607-4.099)$ & 0.35 & * & * \\
\hline Disease duration & $1.003(0.988-1.018)$ & 0.677 & * & * \\
\hline
\end{tabular}

Cl confidence interval, AAV ANCA-associated vasculitis, SLE systemic lupus erythematosus

*Not included in the multivariable model due to the lack of significant association in the univariable analysis

analyzed patient survival to confirm the cut-off value. Consistent with previous reports, patients with other rheumatic diseases tended to have better survival if they received anti-PJP treatment within 6 days of the appearance of the first symptoms (Fig. 3A, 1-year survival rate $87.5 \%$ vs $40 \%, P=0.057)$. However, in $\mathrm{MDA}^{+} \mathrm{DM}$ patients, there was no impact on survival no matter when treatment was started (Fig. $3 \mathrm{~B}, P=0.327$ ). Thus, timely anti-PJP treatment did not benefit MDA5 $5^{+} \mathrm{DM}$ patients and increase their survival in our center.

\section{Discussion}

Early in 1996, a previous study indicated that the prevalence of PJP in systemic lupus erythematosus patients was $1.7 \%$ while in DM patients it was $37.5 \%$, but this was based on a very small cohort $(n=75)$ [14]. Our study is the first to report the incidence of PJP in adult $\mathrm{MDA}^{+} \mathrm{DM}$ patients in an independent cohort and is the largest study so far. For the first time, we describe the different incidences of PJP in anti-MDA5-positive and anti-MDA5-negative IIM patients. The incidence rate in $\mathrm{MDA5}^{+} \mathrm{DM}$ patients is as much as 7.5/100 person-years in our cohort. A Cochrane review recommends that prophylactic treatment should be given when the risk of PJP infection in non-HIV immunocompromised individuals is greater than $6.2 / 100$ person-years [15].

The reason why $\mathrm{MDA}^{+} \mathrm{DM}$ patients are more susceptible to infection by Pneumocystis jirovecii is still unknown. According to previous reports, high risk factors

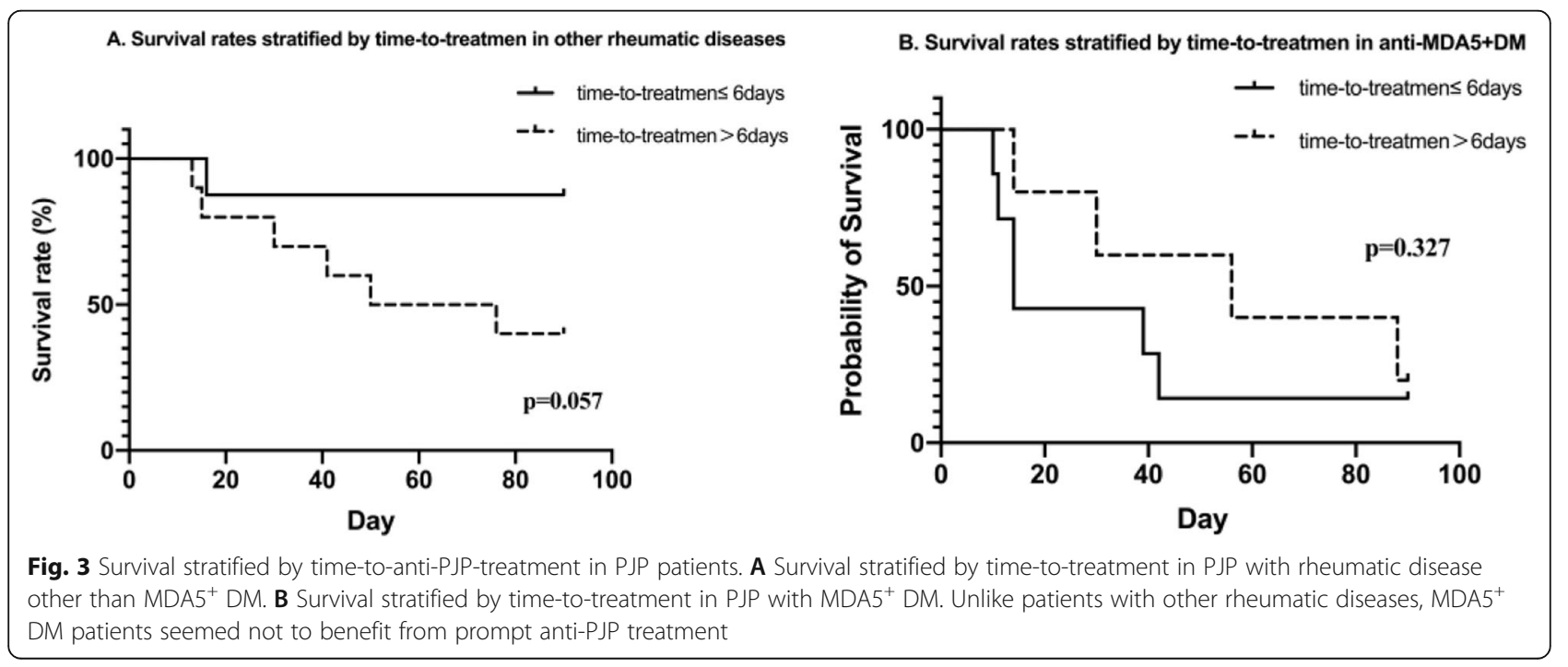


for rheumatic disease complicated with PJP included granulomatosis with polyangiitis; microscopic polyangiitis; autoimmune interstitial pneumonia; use of high-dose glucocorticoids, cyclophosphamide [16], or high-dose methotrexate; being older; having diabetes or nutritional deficiency; and severe lymphocytopenia or low $\mathrm{CD} 4^{+} \mathrm{T}$ cell counts $[17,18]$. Similar to the reports above, comparisons in the present study showed that $\mathrm{PJP}^{+}$IIM patients had been receiving higher doses of corticosteroids and had a higher prevalence of diabetes, lower albumin level, and low $\mathrm{CD}^{+} \mathrm{T}$ cell counts. However, in the multivariate analysis, only the $\mathrm{MDA}^{+} \mathrm{DM}$ disease subtype and low $\mathrm{CD}^{+}{ }^{+} \mathrm{T}$ cell counts were identified as independent risk factors for PJP occurrence. In a comparison of PJP ${ }^{-}$and $\mathrm{PJP}^{+} \mathrm{MDA}^{+} \mathrm{DM}$ patients with similar characteristics and medication, a shorter disease duration prior to infection was noticed, as well as low $\mathrm{CD} 4^{+} \mathrm{T}$ cell counts in infected patients. These findings indicate that we need to pay more attention to PJP in $\mathrm{MDA}^{+} \mathrm{DM}$ patients, especially in the first 3 months after disease onset, or in patients with $\mathrm{CD}^{+} \mathrm{T}$ cell counts of $<200$ cells $/ \mu \mathrm{L}$.

For predicting prognosis, baseline lung involvement has been suggested as a possible factor leading to fatal interstitial pneumonia in $\mathrm{PJP}^{+}$RA patients [19]. Not surprisingly, $\mathrm{CD}^{+} \mathrm{T}$ cell cytopenia predicts mortality in kidney transplant patients with PJP, according to a recent report by Freiwald et al. [20]. Our study reports for the first time that the 3-month mortality rate of adult $\mathrm{MDA}^{+}$DM patients was as high as $83.3 \% . \mathrm{MDA}^{+} \mathrm{DM}$ and $\mathrm{CD} 4^{+} \mathrm{T}$ cell counts were identified by multivariate analysis in our study as independent risk factors for mortality. Given the extremely high prevalence and poor outcome of ILD in MDA5 $5^{+}$DM patients, we speculate that lung comorbidity may contribute to lethality in $\mathrm{PJP}^{+}$ MDA5 ${ }^{+}$DM patients.

One important clinical outcome that was first noticed in our study is that timely PJP treatment did not improve the prognosis of $\mathrm{MDA}^{+} \mathrm{DM}$ patients, which is not the case in other non-HIV PJP infections. Although the number of PJP cases is low in our study, it is alarming enough to encourage us to take measures to deal with such a dangerous condition, in which prophylaxis against PJP may be a good approach [21]. It has been reported that prophylaxis against PJP in rheumatic patients can significantly reduce the incidence of the infection without severe adverse events [22]. The prophylaxis rate was only $4 \%(n=19)$ of patients at admission in our IIM cohort, and merely 43 (9.3\%) patients had been given any continuous anti-PJP prophylaxis since starting treatment for IIM in our hospital. There are currently no recommendation guidelines for PJP management in $\mathrm{MDA}^{+} \mathrm{DM}$ patients. The data presented here suggest the necessity of a further study of PJP prophylaxis in
$\mathrm{MDA5}^{+}$DM patients who may need this on a routine basis.

Limitations of this study include the following. First, the evaluation of PJP occurrence in IIM was observational, a limitation inherent to such studies. Second, the number of PJP cases was rather small, making any inferences from the regression model for independent PJP occurrence factors in IIM or $\mathrm{MDA}^{+} \mathrm{DM}$ potentially less robust. Third, the number of patients on PJP prophylactic treatment was too small for us to assess the benefit of trimethoprim-sulfamethoxazole treatment in $\mathrm{MDA}^{+}$DM patients. Fourth, patients in the IIM cohort were followed up for longer than those with other rheumatic diseases; this may introduce a bias when performing the mortality analysis. Therefore, a randomized controlled trial of PJP prophylaxis is needed.

\section{Conclusions}

Here, we showed that $\mathrm{MDA}^{+} \mathrm{DM}$ patients are highly susceptible to infection with Pneumocystis jirovecii, which is also harder to cure than in other rheumatic diseases. The reason for the higher incidence and mortality may be related to the lower $\mathrm{CD}_{4}^{+} \mathrm{T}$ cell counts and progressive interstitial lung disease in $\mathrm{MDA}^{+}$patients. These findings suggest the necessity for a further systematic study of PJP prophylaxis in $\mathrm{MDA}^{+} \mathrm{DM}$ patients.

\section{Abbreviations}

MDA5 $5^{+}$DM: Anti-melanoma differentiation-associated gene 5 antibodypositive dermatomyositis; MDA5 $5^{-}$IIM: Anti-melanoma differentiationassociated gene 5 antibody-negative idiopathic inflammatory myopathy; PJP: Pneumocystis jirovecii pneumonia; IIM: Idiopathic inflammatory myopathy; PM: Polymyositis; TMP-SMX: Trimethoprim/sulfamethoxazole; IS: Immunosuppressant

\section{Supplementary Information}

The online version contains supplementary material available at https://doi. org/10.1186/s13075-021-02606-8.

Additional file 1: Supplementary figure S1. ROC curve for time-totreatment cut-off valve. A. ROC curve for time-to-treatment cut-off valve in all PJP patients $(P=0.0983)$. B. ROC curve for time-to-treatment cut-off valve in PJP patients without MDA5 ${ }^{+}$DM. Time-to-PJP-treatment cut-off point of 6 days showed $85.7 \%$ sensitivity and $63.6 \%$ specificity and with the Area Under Curve (AUC) $81.2 \%$. The time of six-day was the optimal cut-off point for timely treatment for PJP $(P=0.03)$. C. ROC curve for time-to-treatment cut-off valve in all PJP patients $(P>0.999)$.

Additional file 2: Supplementary table S1. Patients characteristics and PJP infection rate in MDA5 ${ }^{+} \mathrm{DM}$ and $\mathrm{MDA5}^{-}$IIM patients. To show more detail of PJP patient in our cohort.

Additional file 3: Supplementary table S2. Comparison of risk factors in anti-MDA5-ab-positive patients. PJP occurred in a median time of 2 months and with obvious decrease of $C D 4^{+} \mathrm{T}$ cell counts and lymphocytes.

Additional file 4: Supplementary table S3. Characteristics of patients with rheumatic disease and PJP infection from a single center. Compared to other rheumatic disease, PJP with MDA5 $5^{+} \mathrm{DM}$ were characterized as higher percentage of ILD and PJP occurred earlier during the disease duration. 
Additional file 5: Supplementary table S4. Clinical features of the 30 PJP cases at diagnosis. To show more detail of PJP patient in our cohort.

\section{Acknowledgements}

The authors thank Ms Ping Ye and Ms Shuiying Li for the clinical lab technique and follow-up support. The authors would like to express their gratitude to EditSprings (https://www.editsprings.com/) for the expert linguistic services provided.

\section{Authors' contributions}

$\mathrm{LH}, \mathrm{QY}$, and SC participated in the study conception, design, and supervision. $L H, Q F, Y Y, Y L, Q Y$, and SC were involved in the literature search, study design, data collection, statistical analysis, interpretation, and writing and revision of the manuscript. All authors discussed the results and contributed to the final manuscript. The authors read and approved the final manuscript.

\section{Funding}

This work was supported by the National Natural Science Foundation of China (No. 81771752) and the Shanghai Municipal Commission of Health and Family Planning (20204Y0088).

\section{Availability of data and materials}

All data generated or analyzed during this study are included in this published article [and its supplementary information files].

\section{Declarations}

\section{Ethics approval and consent to participate}

The study was in accordance with the Declaration of Helsinki and was approved by the Ethics Committee of Renji Hospital, Shanghai, China (2013216).

\section{Consent for publication}

No individual person's data were presented in any form in this study, and therefore, no consent to publish is required.

\section{Competing interests}

The authors declare that they have no competing interests.

Received: 19 March 2021 Accepted: 14 August 2021

Published online: 04 September 2021

\section{References}

1. Tasaka S. Recent advances in the diagnosis and management of Pneumocystis pneumonia. Tuberc Respir Dis. 2020;83(2):132-40. https://doi. org/10.4046/trd.2020.0015.

2. Hsu H-C, Chang Y-S, Hou T-Y, Chen L-F, Hu L-F, Lin T-M, et al. Pneumocystis jirovecii pneumonia in autoimmune rheumatic diseases: a nationwide population-based study. Clin Rheumatol. 2021 [cited 2021 Jun 19]; Available from: http://link.springer.com/10.1007/s10067-021-05660-4

3. Hsu C-Y, Ko C-H, Wang J-L, Hsu T-C, Lin C-Y. Comparing the burdens of opportunistic infections among patients with systemic rheumatic diseases: a nationally representative cohort study. Arthritis Res Ther. 2019;21(1):211. https://doi.org/10.1186/s13075-019-1997-5.

4. Thomas CF, Limper AH. Pneumocystis pneumonia. N Engl J Med. 2004; 350(24):2487-98. https://doi.org/10.1056/NEJMra032588.

5. Ca M, D S, Ac G, L C-S. Pneumocystis jiroveci pneumonia in rheumatic disease: a 20-year single-centre experience. Clin Exp Rheumatol. 2017;35: 671-673.

6. Khellaf M, Godeau B. Pneumocystis pneumonia among patients with systemic diseases. Presse Médicale. 2009;38(2):251-9. https://doi.org/10.101 6/j.Ipm.2008.11.004.

7. Sabbagh SE, Neely J, Chow A, DeGuzman M, Lai J, Lvovich S, et al. Risk factors associated with Pneumocystis jirovecii pneumonia in juvenile myositis in North America. Rheumatology. 2021;60(2):829-36. https://doi.org/10.1093/ rheumatology/keaa436.

8. Tsuji H, Nakashima R, Hosono Y, Imura Y, Yagita M, Yoshifuji H, et al. Multicenter prospective study of the efficacy and safety of combined immunosuppressive therapy with high-dose glucocorticoid, tacrolimus, and cyclophosphamide in interstitial lung diseases accompanied by anti- melanoma differentiation-associated gene 5-positive dermatomyositis. Arthritis Rheumatol. 2020;72:488-98.

9. Aymonier M, Abed S, Boyé T, Barazzutti H, Fournier B, Morand J-J. Dermatomyositis associated with anti-MDA5 antibodies and pneumocystis pneumonia: two lethal cases. Ann Dermatol Vénéréologie. 2017;144(4):27983. https://doi.org/10.1016/j.annder.2016.09.677.

10. Targoff IN, Miller FW, Medsger TA, Oddis CV. Classification criteria for the idiopathic inflammatory myopathies. Curr Opin Rheumatol. 1997;9(6):52735. https://doi.org/10.1097/00002281-199711000-00008.

11. Kumar SD, Krieger BP. CD4 lymphocyte counts and mortality in AIDS patients requiring mechanical ventilator support due to Pneumocystis carinil pneumonia. Chest. 1998;113(2):430-3. https://doi.org/10.1378/chest.113.2.43 0.

12. Asai N, Motojima S, Ohkuni Y, Matsunuma R, Nakashima K, Iwasaki T, et al. Early diagnosis and treatment are crucial for the survival of Pneumocystis pneumonia patients without human immunodeficiency virus infection. J Infect Chemother. 2012;18(6):898-905. https://doi.org/10.1007/s10156-0120441-4.

13. Ko R-E, Na SJ, Huh K, Suh GY, Jeon K. Association of time-to-treatment with outcomes of Pneumocystis pneumonia with respiratory failure in HIVnegative patients. Respir Res. 2019;20(1):213. https://doi.org/10.1186/s12931019-1188-6.

14. $A K, J O, Y I, H K$. Risk factors for Pneumocystis carinii pneumonia in patients with polymyositis/dermatomyositis or systemic lupus erythematosus. J Rheumatol. 1996;23:1186-8.

15. Stern A, Green H, Paul M, Vidal L, Leibovici L. Prophylaxis for Pneumocystis pneumonia (PJP) in non-HIV immunocompromised patients. Cochrane Gynaecological, Neuro-oncology and Orphan Cancer Group, editor. Cochrane Database Syst Rev. 2014 [cited 2021 Mar 10]; Available from: http://doi.wiley.com/10.1002/14651858.CD005590.pub3

16. Zhang Y, Zheng Y. Pneumocystis jirovecii pneumonia in mycophenolate mofetil-treated patients with connective tissue disease: analysis of 17 cases. Rheumatol Int. 2014;34(12):1765-71. https://doi.org/10.1007/s00296-014-3 073-4.

17. Wolfe RM, Peacock JE. Pneumocystis pneumonia and the rheumatologist: which patients are at risk and how can PJP be prevented? Curr Rheumatol Rep. 2017;19(6):35. https://doi.org/10.1007/s11926-017-0664-6.

18. Herrou J, De Lastours V. Predictive factors of pneumocystis pneumonia in patients with rheumatic diseases exposed to prolonged high-dose glucocorticoids. Ann Rheum Dis. 2020;79(2):e23. https://doi.org/10.1136/a nnrheumdis-2018-214718.

19. Yoshida Y, Takahashi Y, Minemura N, Ueda Y, Yamashita H, Kaneko H, et al. Prognosis of pneumocystis pneumonia complicated in patients with rheumatoid arthritis (RA) and non-RA rheumatic diseases. Mod Rheumatol. 2012;22:509-14.

20. Freiwald T, Büttner S, Cheru NT, Avaniadi D, Martin SS, Stephan C, et al. CD4(+) T cell lymphopenia predicts mortality from Pneumocystis pneumonia in kidney transplant patients. Clin Transplant. 2020;34:1-10.

21. Schmajuk G, Jafri K, Evans M, Shiboski S, Gianfrancesco M, Izadi Z, et al. Pneumocystis jirovecii pneumonia (PJP) prophylaxis patterns among patients with rheumatic diseases receiving high-risk immunosuppressant drugs. Semin Arthritis Rheum. 2019;48:1087-92.

22. Park JW, Curtis JR, Moon J, Song YW, Kim S, Lee EB. Prophylactic effect of trimethoprim-sulfamethoxazole for pneumocystis pneumonia in patients with rheumatic diseases exposed to prolonged high-dose glucocorticoids. Ann Rheum Dis. 2018;77:644-9.

\section{Publisher's Note}

Springer Nature remains neutral with regard to jurisdictional claims in published maps and institutional affiliations. 